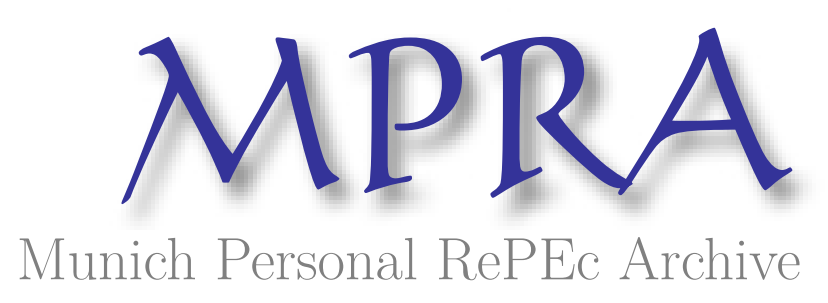

Mathematical model of the Greek crisis

Krouglov, Alexei

14 August 2015

Online at https://mpra.ub.uni-muenchen.de/66115/

MPRA Paper No. 66115, posted 14 Aug 2015 20:00 UTC 


\title{
Mathematical Model of the Greek Crisis
}

\author{
Alexei Krouglov
}

alexkrouglov@gmail.com 


\title{
Mathematical Model of the Greek Crisis
}

\begin{abstract}
Presented is a simplified mathematical model of the Greek economy with a reduced description of different stages of the current Greek crisis. Explored are conditions where a stream of investments can pull economy from the crisis. It has been theoretically proven that an investment in the benign conditions where demand is sustained produces higher nominal economic growth than an investment in the austere conditions where demand is cut.
\end{abstract}

JEL Classification Numbers: E32, G01, E22

Keywords: Greek crisis, investment, modeling 


\section{Introduction}

Greek economy is in a dire economic recession. There are few factors that have brought Greece into the crisis and are preventing the recovery. They include (i) debt burden and corresponding necessity to service the debt, (ii) the lack of investment inducing an economic growth, and (iii) an import of (cheaper and better quality) consumer goods from abroad stifling the local production.

Here I present a simplified mathematical model describing the four consecutive stages of the Greek crisis (before the Eurozone, before the Euro crisis, after the Euro crisis, and during the austerity) and two possible recovery phases (corresponding to austere reforms and benign reforms).

I describe a mathematical model of the market of single-product economy, which can give us an explicit observation of interactions among different economic variables. Economic forces acting on the market represent inherent market forces of demand and supply complemented with the forces caused by an investment, an external supply of product on the market, debt and corresponding debt servicing. The market actions are expressed through the system of ordinary differential equations.

The model uses recent results for a nominal economic growth and decline performed in (Krouglov, 2014), previous research of a credit expansion and contraction in (Krouglov, 2015a), and an impact of the debt and investment on economic growth in (Krouglov, 2015b).

\section{Mathematical Model of the Greek Crisis}

Concepts and methodology presented in this paper are based on the framework of mathematical dynamics of economic systems developed in (Krouglov, 2006; 2009). When there are no disturbing economic forces, the market is in equilibrium position, i.e., the supply of and demand for product are equal and are developing with a constant rate and a price of the product is fixed. When the balance between the volumes 
of product supply and demand is broken, market is experiencing economic forces, which act to bring the market to a new equilibrium position.

The framework was extended with new results. Conditions for a nominal economic growth and decline were amply investigated in (Krouglov, 2014). Questions of the credit expansion and contraction with regard to an economic growth were investigated in (Krouglov, 2015a). A mutual impact of the debt and investment on economic growth was explored in (Krouglov, 2015b).

These theoretical results will be utilized to examine a simplified mathematical model of Greek crisis.

\subsection{Model of Greek Economy prior to the Eurozone}

We can consider that during that period Greek economy didn't have a robust economic growth due to the lack of significant investments. Also the amount of debt was not a problem for the economy.

According to this scenario, the amount of debt was equal to $S_{D}(t)=0$ prior to the time $t<t_{0}$. Likewise, the accumulated amount of debt servicing cost was equal to $S_{S}(t)=0$ prior to the time $t<t_{0}$. Also, the amount of investment was equal to $S_{I}(t)=0$ prior to the time $t<t_{0}$.

Thus, there were no disturbing economic forces, the market was in equilibrium position, i.e., the supply of and demand for product were equal, they were developing with a constant rate and a price of the product was fixed (Krouglov, 2006; 2009). 
We assume the market had been in an equilibrium until time $t=t_{0}$, volumes of the product supply $V_{S}(t)$ and demand $V_{D}(t)$ on market were equal, and they both were developing with a constant rate $r_{D}^{0}$. The product price $P(t)$ at that time was fixed,

$V_{D}(t)=r_{D}^{0}\left(t-t_{0}\right)+V_{D}^{0}$

$V_{S}(t)=V_{D}(t)$

$P(t)=P^{0}$

where $V_{D}\left(t_{0}\right)=V_{D}^{0}$.

We can evaluate a rate of nominal demand $E_{D}(t) \equiv P(t) \times r_{D}(t)$ for the product in this period where $r_{D}(t) \equiv \frac{d V_{D}(t)}{d t}$. It takes place, $E_{D}(t)={ }_{0} E_{D}$ where ${ }_{0} E_{D} \equiv P^{0} r_{D}^{0}$ until time $t=t_{0}$.

We see that the economy in this period was at the undisturbed state and this economic development could last for a long term.

\subsection{Model of Greek Economy prior to Euro Crisis}

After Greece joined the Eurozone (at the time $t=t_{0}$ ) it took an advantage of its new status and increased the demand by taking on a significant amount of debt. The increased demand was met through importing consumer goods while the Greek economy was still experiencing a lack of investments. The debt servicing cost was not a problem for the economy (since an interest rate was low and a new loan could be used to pay for the old one). 
According to the scenario, the amount of debt was equal to $S_{D}(t)=\delta_{D}\left(t-t_{0}\right)$ where $\delta_{D}>0$ at the time $t \geq t_{0}$. Alike, the accumulated amount of debt servicing cost was equal to $S_{S}(t) \approx 0$ at the time $t \geq t_{0}$. The amount of external supply of product was equal to $S_{X}(t)=\delta_{X}\left(t-t_{0}\right)$ where $\delta_{X}>0$ and $\delta_{X}=\delta_{D}$ at the time $t \geq t_{0}$. Also, the amount of investment was equal to $S_{I}(t)=0$ at the time $t \geq t_{0}$.

Thus, the market was still in equilibrium position, i.e., both the expanded supply of and expanded demand for product were equal, they were developing with a constant rate and the price of product was fixed.

At the time $t \geq t_{0}$, the volumes of product's expanded supply $\hat{V}_{S}(t)$ and expanded demand $\hat{V}_{D}(t)$ were equal, and they both were developing with a constant rate $r_{D}^{0}+\delta_{D}$. The product price $P(t)$ at that time was fixed,

$\hat{V}_{D}(t)=r_{D}^{0}\left(t-t_{0}\right)+\delta_{D}\left(t-t_{0}\right)+V_{D}^{0}$

$\hat{V}_{S}(t)=r_{D}^{0}\left(t-t_{0}\right)+\delta_{X}\left(t-t_{0}\right)+V_{D}^{0}$

$P(t)=P^{0}$

where $V_{D}\left(t_{0}\right)=V_{D}^{0}$.

Hence, it takes place,

$\hat{V}_{D}(t)=\hat{V}_{S}(t)$

We can evaluate a rate of nominal expanded demand $\hat{E}_{D}(t) \equiv P(t) \times \hat{r}_{D}(t)$ for the product in this period where $\hat{r}_{D}(t) \equiv \frac{d \hat{V}_{D}(t)}{d t}$. It takes place, $\hat{E}_{D}(t)=E_{1} E_{D}$ where ${ }_{1} E_{D} \equiv P^{0}\left(r_{D}^{0}+\delta_{D}\right)$ or ${ }_{1} E_{D}={ }_{0} E_{D}+P^{0} \delta_{D}$ at the time $t \geq t_{0}$, i.e., the rate of nominal expanded demand had increased after Greece joined the Eurozone. 
We see that prosperity of the Greeks had increased when the country joined the Eurozone, its economy in this period was at the equilibrium state and this economic situation could last for a long time until Greece could keep the trust of capital markets.

\section{3. $\quad$ Model of Greek Economy after Euro Crisis}

After the European debt crisis (at the time $t=t_{1} \geq t_{0}$ ) an interest rate for the Greek debt had dramatically increased. Greece couldn't borrow anymore from the capital market. Debt servicing cost became a huge problem for the Greek economy. At the same time Greece couldn't use debt anymore as a tool to increase the demand. It couldn't also pay for the imported consumer goods.

According to the scenario, the amount of debt borrowed from the capital market was frozen $S_{D}(t)={ }_{1} S_{D}$ at the time $t \geq t_{1}$. Thus, the previous debt wouldn't be able to be repaid and would be accumulated. The latter would cause the rate of debt servicing cost to increase and the accumulated amount of debt servicing cost to

accelerate $S_{S}(t)=\delta_{S}\left(t-t_{1}\right)+\frac{\varepsilon_{S}}{2}\left(t-t_{1}\right)^{2}$ where $\delta_{S} \geq 0, \varepsilon_{S}>0$ at the time $t \geq t_{1}$. The amount of external supply of product would be frozen $S_{X}(t)={ }_{1} S_{X}$ where ${ }_{1} S_{X}={ }_{1} S_{D}$ at the time $t \geq t_{1}$. Also, the amount of investment would be equal to $S_{I}(t)=0$ at the time $t \geq t_{1}$.

Thus, the balance between the volumes of product's expanded supply and expanded demand was broken. The market was experiencing economic forces, which were acting to bring the market to a new equilibrium.

Economic forces trying to bring the market into a new equilibrium position are described by the following ordinary differential equations with regard to the volumes of product supply $V_{S}(t)$, demand $V_{D}(t)$, and 
price $P(t)$ given the accumulated amounts of debt $S_{D}(t)$, debt servicing cost $S_{S}(t)$, and external supply of product $S_{X}(t)$

$$
\begin{aligned}
& \frac{d P(t)}{d t}=-\lambda_{P}\left(V_{S}(t)+S_{X}(t)-V_{D}(t)-S_{D}(t)+S_{S}(t)\right) \\
& \frac{d^{2} V_{S}(t)}{d t^{2}}=\lambda_{S} \frac{d P(t)}{d t} \\
& \frac{d^{2} V_{D}(t)}{d t^{2}}=-\lambda_{D} \frac{d^{2} P(t)}{d t^{2}}
\end{aligned}
$$

In Eqs. (8) - (10) above the values $\lambda_{P}, \lambda_{S}, \lambda_{D} \geq 0$ are constants and they characterize price inertness, supply inducement, and demand amortization correspondingly.

Solution of the system of equations similar to the Eqs. (8) - (10) can be found as shown in (Krouglov, 2006; 2009) using a conventional technique (Piskunov, 1965; Petrovski, 1966).

It takes place for the product's price $P(t)$, demand $V_{D}(t)$, and supply $V_{S}(t)$ when $t \rightarrow+\infty$,

$$
\begin{aligned}
& P(t) \rightarrow-\frac{\varepsilon_{S}}{\lambda_{S}}\left(t-t_{1}\right)+P^{0}-\frac{\delta_{S}}{\lambda_{S}}+\frac{\lambda_{D}}{\lambda_{S}^{2}} \varepsilon_{S} \\
& V_{D}(t) \rightarrow\left(r_{D}^{0}+\frac{\lambda_{D}}{\lambda_{S}} \varepsilon_{S}\right)\left(t-t_{1}\right)+V_{D}^{0}+\frac{\lambda_{D}}{\lambda_{S}} \delta_{S}-\frac{\lambda_{D}^{2}}{\lambda_{S}^{2}} \varepsilon_{S} \\
& V_{S}(t) \rightarrow\left(r_{D}^{0}-\delta_{S}+\frac{\lambda_{D}}{\lambda_{S}} \varepsilon_{S}\right)\left(t-t_{1}\right)-\frac{\varepsilon_{S}}{2}\left(t-t_{1}\right)^{2}+V_{D}^{0}+\frac{\lambda_{D}}{\lambda_{S}} \delta_{S}+\frac{\varepsilon_{S}}{\lambda_{P} \lambda_{S}}-\frac{\lambda_{D}^{2}}{\lambda_{S}^{2}} \varepsilon_{S}
\end{aligned}
$$

It also takes place for the product's expanded demand $\hat{V}_{D}(t)=V_{D}(t)+S_{D}(t)-S_{S}(t)$ and the product's expanded supply $\hat{V}_{S}(t)=V_{S}(t)+S_{X}(t)$ when $t \rightarrow+\infty$,

$$
\hat{V}_{D}(t) \rightarrow\left(r_{D}^{0}-\delta_{S}+\frac{\lambda_{D}}{\lambda_{S}} \varepsilon_{S}\right)\left(t-t_{1}\right)-\frac{\varepsilon_{S}}{2}\left(t-t_{1}\right)^{2}+V_{D}^{0}+{ }_{1} S_{D}+\frac{\lambda_{D}}{\lambda_{S}} \delta_{S}-\frac{\lambda_{D}^{2}}{\lambda_{S}^{2}} \varepsilon_{S}
$$


$\hat{V}_{S}(t) \rightarrow\left(r_{D}^{0}-\delta_{S}+\frac{\lambda_{D}}{\lambda_{S}} \varepsilon_{S}\right)\left(t-t_{1}\right)-\frac{\varepsilon_{S}}{2}\left(t-t_{1}\right)^{2}+V_{D}^{0}+{ }_{1} S_{X}+\frac{\lambda_{D}}{\lambda_{S}} \delta_{S}+\frac{\varepsilon_{S}}{\lambda_{P} \lambda_{S}}-\frac{\lambda_{D}^{2}}{\lambda_{S}^{2}} \varepsilon_{S}$

We can observe that debt servicing cost would violate the equilibrium position. It would eventually exceed the available demand since the expanded demand $\hat{V}_{D}(t) \rightarrow-\infty$ when $t \rightarrow+\infty$ according to Eq. (14). We can also see that debt servicing cost would cause a deflation forcing the price to decrease $P(t) \rightarrow-\infty$ when $t \rightarrow+\infty$ according to Eq. (11).

We can also evaluate a rate of nominal demand $E_{D}(t) \equiv P(t) \times r_{D}(t)$ for the product in this period. ${ }^{1}$ It takes place, $E_{D}(t) \rightarrow\left(-\frac{\varepsilon_{S}}{\lambda_{S}}\left(t-t_{1}\right)+P^{0}-\frac{\delta_{S}}{\lambda_{S}}+\frac{\lambda_{D}}{\lambda_{S}^{2}} \varepsilon_{S}\right)\left(r_{D}^{0}+\frac{\lambda_{D}}{\lambda_{S}} \varepsilon_{S}\right)$ when $t \rightarrow+\infty$.

Thus, if the debt servicing cost $S_{S}(t)$ increases with a constant acceleration $\varepsilon_{S}>0$ then the value of acceleration $\varepsilon_{S}$ would cause an unrestricted decrease of the rate of nominal demand $E_{D}(t)$. The rate of nominal demand $E_{D}(t)$ would be continuously decreasing with the passage of time.

We can estimate a change $e_{D}(t)$ in the rate of nominal demand $E_{D}(t)$ where $e_{D}(t) \equiv \frac{d E_{D}(t)}{d t}$, i.e., the change in rate of nominal demand, which roughly represents the change in the product earning on market.

\footnotetext{
${ }^{1}$ Since both the price $P(t) \rightarrow-\infty$ and the rate of expanded demand $\hat{r}_{D}(t) \rightarrow-\infty$, the rate of nominal expanded demand $\hat{E}_{D}(t) \rightarrow+\infty$. In practice, it is better to restrict the price $P(t) \geq 0$ to avoid confusing conclusions.
} 
It takes place for $t \rightarrow+\infty, e_{D}(t) \rightarrow-\frac{\varepsilon_{S}}{\lambda_{S}}\left(r_{D}^{0}+\frac{\lambda_{D}}{\lambda_{S}} \varepsilon_{S}\right)<0$ when $\varepsilon_{S}>0$. Therefore, the variable $e_{D}(t)$ is always negative in the region $\varepsilon_{S}>0$. It causes in the region $\varepsilon_{S}>0$ an unrestricted decrease of the rate of nominal demand $E_{D}(t)$ with a constant deceleration $-\frac{\varepsilon_{S}}{\lambda_{S}}\left(r_{D}^{0}+\frac{\lambda_{D}}{\lambda_{S}} \varepsilon_{S}\right)<0$.

We can see that Greek economy was in a state of free fall after the Euro crisis. The accelerated pace of debt servicing cost would cause a continuous price fall (a.k.a. deflation). The rate and the amount of debt servicing cost could eventually exceed the rate and the volume of demand for the product. The rate of nominal demand would be monotonically decreasing with a constant deceleration. Clearly, such economic situation could not last for a long time.

\subsection{Model of Greek Economy at Austerity}

To rectify the economic situation the Eurozone countries helped Greece to restructure its debt (at the time $t=t_{2} \geq t_{1}$ ). Greece couldn't still borrow from the capital market but the existing debt was reorganized. The debt servicing cost in big part was alleviated. Also, Greece couldn't use debt to increase the demand while Greece had to continue importing the consumer goods at the request of Eurozone countries.

Also, we assume that $t_{2}=t_{1}+\Delta$ where $\Delta \approx 0$ or $t_{2} \approx t_{1}$ in order to avoid accounting for economic deviation during the time interval $t_{1} \leq t<t_{2}$, which otherwise would obscure the calculations excessively.

According to the scenario, the amount of debt borrowed from capital market was frozen $S_{D}(t)={ }_{1} S_{D}$ at the time $t \geq t_{2}$. Alike, the accumulated amount of debt servicing cost was frozen and equal to $S_{S}(t) \approx 0$ at the time $t \geq t_{2}$ (since $t_{2} \approx t_{1}$ ). Likewise, the amount of external supply of product on the market was 
equal to $S_{X}(t)={ }_{1} S_{X}+\delta_{X}\left(t-t_{2}\right)$ where ${ }_{1} S_{X}={ }_{1} S_{D}, \delta_{X}>0$ at the time $t \geq t_{2}$. Also, the amount of investment was equal to $S_{I}(t)=0$ at the time $t \geq t_{2}$.

Thus, the balance between the volumes of product's expanded supply and expanded demand was broken. The market was experiencing economic forces, which were acting to bring the market to a new equilibrium.

Economic forces trying to bring the market into a new equilibrium position are described by the following ordinary differential equations with regard to the volumes of product supply $V_{S}(t)$, demand $V_{D}(t)$, and price $P(t)$ given the accumulated amounts of debt $S_{D}(t)$, debt servicing cost $S_{S}(t)$, and external supply of product $S_{X}(t)$

$$
\begin{aligned}
& \frac{d P(t)}{d t}=-\lambda_{P}\left(V_{S}(t)+S_{X}(t)-V_{D}(t)-S_{D}(t)\right) \\
& \frac{d^{2} V_{S}(t)}{d t^{2}}=\lambda_{S} \frac{d P(t)}{d t} \\
& \frac{d^{2} V_{D}(t)}{d t^{2}}=-\lambda_{D} \frac{d^{2} P(t)}{d t^{2}}
\end{aligned}
$$

It takes place for the product's price $P(t)$, demand $V_{D}(t)$, and supply $V_{S}(t)$ when $t \rightarrow+\infty$,

$$
\begin{aligned}
& P(t) \rightarrow P^{0}-\frac{\delta_{X}}{\lambda_{S}} \\
& V_{D}(t) \rightarrow r_{D}^{0}\left(t-t_{2}\right)+V_{D}^{0}+\frac{\lambda_{D}}{\lambda_{S}} \delta_{X} \\
& V_{S}(t) \rightarrow\left(r_{D}^{0}-\delta_{X}\right)\left(t-t_{2}\right)+V_{D}^{0}+\frac{\lambda_{D}}{\lambda_{S}} \delta_{X}
\end{aligned}
$$


It also takes place for the product's expanded demand $\hat{V}_{D}(t)=V_{D}(t)+S_{D}(t)$ and the product's expanded supply $\hat{V}_{S}(t)=V_{S}(t)+S_{X}(t)$ when $t \rightarrow+\infty$,

$\hat{V}_{D}(t) \rightarrow r_{D}^{0}\left(t-t_{2}\right)+V_{D}^{0}+{ }_{1} S_{D}+\frac{\lambda_{D}}{\lambda_{S}} \delta_{X}$

$\hat{V}_{S}(t) \rightarrow r_{D}^{0}\left(t-t_{2}\right)+V_{D}^{0}+{ }_{1} S_{X}+\frac{\lambda_{D}}{\lambda_{S}} \delta_{X}$

We can assess a rate of nominal expanded demand $\hat{E}_{D}(t) \equiv P(t) \times \hat{r}_{D}(t)$ at the time $t \geq t_{2}$. It takes place,

$\hat{E}_{D}(t) \rightarrow\left(P^{0}-\frac{\delta_{X}}{\lambda_{S}}\right) r_{D}^{0}$ when $t \rightarrow+\infty$. We can compare ${ }_{2} E_{D} \equiv\left(P^{0}-\frac{\delta_{X}}{\lambda_{S}}\right) r_{D}^{0}$ with a rate of

nominal demand ${ }_{0} E_{D} \equiv P^{0} r_{D}^{0}$ encountered by Greece before the Eurozone. We can observe

${ }_{2} E_{D}={ }_{0} E_{D}-\frac{\delta_{X}}{\lambda_{S}} r_{D}^{0}$, i.e., the long-term rate of nominal demand fell down after joining the Eurozone.

Thus, we can see that austerity measures and import of consumer goods from other countries brought Greek economy to the situation worse than prior of joining the Eurozone. This economic situation could last for a long time if only the Greeks keep their patience.

Note that the external supply of product caused the prices to fall (i.e., created deflation). When the external supply of product is furnished with a constant rate, the effect is a restricted long-term nominal economic decline (a.k.a. secular stagnation), which was amply described in (Krouglov, 2014).

\section{Mathematical Model of Economic Reforms}

I present in this section research what might constitute a recovery for the Greek economy. 
According to (Krouglov, 2014), a nominal economic growth is fundamentally driven (among other factors) by the continuous flow of investment.

If investment of the product is performed with a constant rate the result is a limited long-term nominal economic growth. If investment of the product is done with a modest constant acceleration the result is an unlimited long-term nominal economic growth. If investment of the product is done with a large constant acceleration the result is an unlimited long-term nominal economic decline.

Let me show how a constant-rate investment of product can benefit the Greek economy. I will explore two options: the first one where demand is cut due to the austerity measures; and the second one where demand is sustained to alleviate the economic hardship.

\subsection{Model of Austere Economic Reforms}

Assume we are at the time $t=t_{3} \geq t_{2}$ ). In order to experience an economic growth the Greek economy has to utilize investments (Krouglov, 2014). The Eurozone countries promised to help Greece with handling its debt problem. However, they don't allow Greece to use debt to increase the demand and want Greece to continue importing the consumer goods from them.

Again, we assume that $t_{3}=t_{2}+\Delta$ where $\Delta \approx 0$ or $t_{3} \approx t_{2}$ in order to avoid accounting for the economic deviation during the time interval $t_{2} \leq t<t_{3}$ for the same reason as earlier.

According to the scenario, the amount of debt borrowed from the capital market was frozen $S_{D}(t)={ }_{1} S_{D}$ at the time $t \geq t_{3}$. Alike, the accumulated amount of debt servicing cost was frozen and equal to $S_{S}(t) \approx 0$ at the time $t \geq t_{3}$ (since $t_{3} \approx t_{2}$ ). The amount of external supply of product on the market was equal to 
$S_{X}(t)={ }_{1} S_{X}+\delta_{X}\left(t-t_{3}\right)$ where ${ }_{1} S_{X}={ }_{1} S_{D}, \delta_{X}>0$ at the time $t \geq t_{3}$. Also, the amount of investment was equal to $S_{I}(t)=\delta_{I}\left(t-t_{3}\right)$ where $\delta_{I}>0$ at the time $t \geq t_{3}$.

For the sake of simplicity, we assume $\delta_{X}=\delta_{I}$ at the time $t \geq t_{3}$ (later we will remove this assumption).

Thus, the market is still in an equilibrium position, i.e., both the expanded supply of and expanded demand for product are equal, they are developing with a constant rate and a price of the product is fixed.

At the time $t \geq t_{3}$, the volumes of product's expanded supply $\hat{V}_{S}(t)=V_{S}(t)-S_{I}(t)+S_{X}(t)$ and expanded demand $\hat{V}_{D}(t)=V_{D}(t)+S_{D}(t)$ are equal, and they are developing with a constant rate $r_{D}^{0}$. The product price $P(t)$ at that time is fixed,

$\hat{V}_{D}(t)=r_{D}^{0}\left(t-t_{3}\right)+V_{D}^{0}+{ }_{1} S_{D}$

$\hat{V}_{S}(t)=r_{D}^{0}\left(t-t_{3}\right)-\delta_{I}\left(t-t_{3}\right)+\delta_{X}\left(t-t_{3}\right)+V_{D}^{0}+{ }_{1} S_{X}$

$P(t)=P^{0}$

where $V_{D}\left(t_{3}\right)=V_{D}^{0}$.

Therefore, it takes place,

$\hat{V}_{D}(t)=\hat{V}_{S}(t)$

We can evaluate a rate of nominal expanded demand $\hat{E}_{D}(t) \equiv P(t) \times \hat{r}_{D}(t)$ for the product in this period.

It takes place, $\hat{E}_{D}(t)={ }_{3} E_{D}$ where ${ }_{3} E_{D} \equiv P^{0} r_{D}^{0}$ at the time $t \geq t_{3}$, i.e., the rate of nominal expanded demand doesn't change ${ }_{3} E_{D}={ }_{0} E_{D}$ from prior to the Eurozone. The results of economic reforms don't look impressive. The economic situation could last for a long time if only the Greeks keep their patience. 
Let us now assume that $\delta_{I}>\delta_{X}>0$ at the time $t \geq t_{3}$, which causes $\hat{V}_{D}(t)>\hat{V}_{S}(t)$ just after the time $t=t_{3}$.

Thus, the balance between the volumes of product's expanded supply and expanded demand is broken. The market is experiencing economic forces, which are acting to bring the market to a new equilibrium.

It takes place for the product's price $P(t)$, demand $V_{D}(t)$, and supply $V_{S}(t)$ when $t \rightarrow+\infty$,

$$
\begin{aligned}
& P(t) \rightarrow P^{0}+\frac{\delta_{I}-\delta_{X}}{\lambda_{S}} \\
& V_{D}(t) \rightarrow r_{D}^{0}\left(t-t_{3}\right)+V_{D}^{0}-\frac{\lambda_{D}}{\lambda_{S}}\left(\delta_{I}-\delta_{X}\right) \\
& V_{S}(t) \rightarrow\left(r_{D}^{0}+\delta_{I}-\delta_{X}\right)\left(t-t_{3}\right)+V_{D}^{0}-\frac{\lambda_{D}}{\lambda_{S}}\left(\delta_{I}-\delta_{X}\right)
\end{aligned}
$$

It also takes place for the product's expanded demand $\hat{V}_{D}(t)=V_{D}(t)+S_{D}(t)$ and the product's expanded supply $\hat{V}_{S}(t)=V_{S}(t)+S_{X}(t)-S_{I}(t)$ when $t \rightarrow+\infty$,

$$
\begin{aligned}
& \hat{V}_{D}(t) \rightarrow r_{D}^{0}\left(t-t_{3}\right)+V_{D}^{0}+{ }_{1} S_{D}-\frac{\lambda_{D}}{\lambda_{S}}\left(\delta_{I}-\delta_{X}\right) \\
& \hat{V}_{S}(t) \rightarrow r_{D}^{0}\left(t-t_{3}\right)+V_{D}^{0}+{ }_{1} S_{X}-\frac{\lambda_{D}}{\lambda_{S}}\left(\delta_{I}-\delta_{X}\right)
\end{aligned}
$$

We value a rate of nominal expanded demand $\hat{E}_{D}(t) \equiv P(t) \times \hat{r}_{D}(t)$ at the time $t \geq t_{3}$. It takes place, $\hat{E}_{D}(t) \rightarrow\left(P^{0}+\frac{\delta_{I}-\delta_{X}}{\lambda_{S}}\right) r_{D}^{0}$ when $t \rightarrow+\infty$. We compare ${ }_{3} E_{D}^{+} \equiv\left(P^{0}+\frac{\delta_{I}-\delta_{X}}{\lambda_{S}}\right) r_{D}^{0}$ with a rate of nominal demand ${ }_{0} E_{D} \equiv P^{0} r_{D}^{0}$ experienced before the Eurozone. We observe ${ }_{3} E_{D}^{+}={ }_{0} E_{D}+\frac{\delta_{I}-\delta_{X}}{\lambda_{S}} r_{D}^{0}$, 
i.e., the long-term rate of nominal demand increases compared to one before the Eurozone. The economic reforms do bring positive results here. The economic situation could last for a long time.

Let us now assume that $\delta_{X}>\delta_{I}>0$ at the time $t \geq t_{3}$, which causes $\hat{V}_{D}(t)<\hat{V}_{S}(t)$ just after the time $t=t_{3}$.

We estimate a rate of nominal demand $\hat{E}_{D}(t) \equiv P(t) \times \hat{r}_{D}(t)$ at the time $t \geq t_{3}$. When $t \rightarrow+\infty$ it takes place, $\hat{E}_{D}(t) \rightarrow\left(P^{0}-\frac{\delta_{X}-\delta_{I}}{\lambda_{S}}\right) r_{D}^{0}$. We compare ${ }_{3} E_{D}^{-} \equiv\left(P^{0}-\frac{\delta_{X}-\delta_{I}}{\lambda_{S}}\right) r_{D}^{0}$ with the rate of nominal demand ${ }_{0} E_{D} \equiv P^{0} r_{D}^{0}$ experienced before the Eurozone. We see ${ }_{3} E_{D}^{-}={ }_{0} E_{D}-\frac{\delta_{X}-\delta_{I}}{\lambda_{S}} r_{D}^{0}$, i.e., the longterm rate of nominal demand decreases compared to one before the Eurozone. The economic reforms bring negative results here. The economic situation could last for a long time if only people keep their patience.

\subsection{Model of Benign Economic Reforms}

We are still at the time $t=t_{3} \geq t_{2}$ ). Assume a hypothetical situation that Eurozone countries allow Greece to increase demand by using debt. The Eurozone countries continue helping Greece with handling the debt. Greece continues importing the consumer goods. The economy makes investments for economic growth.

Again, we assume that $t_{3}=t_{2}+\Delta$ where $\Delta \approx 0$ or $t_{3} \approx t_{2}$.

According to the scenario, the amount of debt is equal to $S_{D}(t)={ }_{1} S_{D}+\delta_{D}\left(t-t_{3}\right)$ where $\delta_{D}>0$ at the time $t \geq t_{3}$. The accumulated amount of debt servicing cost is frozen and equal to $S_{S}(t) \approx 0$ at the time 
$t \geq t_{3}$. The amount of external supply of product on the market is equal to $S_{X}(t)={ }_{1} S_{X}+\delta_{X}\left(t-t_{3}\right)$ where ${ }_{1} S_{X}={ }_{1} S_{D}, \delta_{X}>0$ at the time $t \geq t_{3}$. We assume $\delta_{X}=\delta_{D}$. The amount of investment is equal to $S_{I}(t)=\delta_{I}\left(t-t_{3}\right)$ where $\delta_{I}>0$ at the time $t \geq t_{3}$.

Thus, the balance between the volumes of product's expanded supply and expanded demand is broken. The market is experiencing economic forces, which are acting to bring the market to a new equilibrium.

Economic forces trying to bring the market into a new equilibrium position are described by the following ordinary differential equations with regard to the volumes of product supply $V_{S}(t)$, demand $V_{D}(t)$, and price $P(t)$ given the accumulated amounts of debt $S_{D}(t)$, debt servicing cost $S_{S}(t)$, investment $S_{I}(t)$, and external supply of product $S_{X}(t)$,

$$
\begin{aligned}
& \frac{d P(t)}{d t}=-\lambda_{P}\left(V_{S}(t)-V_{D}(t)-S_{D}(t)-S_{I}(t)+S_{X}(t)\right) \\
& \frac{d^{2} V_{S}(t)}{d t^{2}}=\lambda_{S} \frac{d P(t)}{d t} \\
& \frac{d^{2} V_{D}(t)}{d t^{2}}=-\lambda_{D} \frac{d^{2} P(t)}{d t^{2}}
\end{aligned}
$$

It takes place for the product's price $P(t)$, demand $V_{D}(t)$, and supply $V_{S}(t)$ when $t \rightarrow+\infty$,

$$
\begin{aligned}
& P(t) \rightarrow P^{0}+\frac{\delta_{I}}{\lambda_{S}} \\
& V_{D}(t) \rightarrow r_{D}^{0}\left(t-t_{3}\right)+V_{D}^{0}-\frac{\lambda_{D}}{\lambda_{S}} \delta_{I} \\
& V_{S}(t) \rightarrow\left(r_{D}^{0}+\delta_{I}\right)\left(t-t_{3}\right)+V_{D}^{0}-\frac{\lambda_{D}}{\lambda_{S}} \delta_{I}
\end{aligned}
$$


It also takes place for the product's expanded demand $\hat{V}_{D}(t)=V_{D}(t)+S_{D}(t)$ and the product's expanded supply $\hat{V}_{S}(t)=V_{S}(t)+S_{X}(t)-S_{I}(t)$ when $t \rightarrow+\infty$,

$\hat{V}_{D}(t) \rightarrow\left(r_{D}^{0}+\delta_{D}\right)\left(t-t_{3}\right)+V_{D}^{0}+{ }_{1} S_{D}-\frac{\lambda_{D}}{\lambda_{S}} \delta_{I}$

$\hat{V}_{S}(t) \rightarrow\left(r_{D}^{0}+\delta_{X}\right)\left(t-t_{3}\right)+V_{D}^{0}+{ }_{1} S_{X}-\frac{\lambda_{D}}{\lambda_{S}} \delta_{I}$

We can assess a rate of nominal demand $\hat{E}_{D}(t) \equiv P(t) \times \hat{r}_{D}(t)$ at the time $t \geq t_{2}$. When $t \rightarrow+\infty$ it takes place, $\hat{E}_{D}(t) \rightarrow\left(P^{0}+\frac{\delta_{I}}{\lambda_{S}}\right)\left(r_{D}^{0}+\delta_{D}\right)$. We compare ${ }_{3} E_{D}^{++} \equiv\left(P^{0}+\frac{\delta_{I}}{\lambda_{S}}\right)\left(r_{D}^{0}+\delta_{D}\right)$ with the rate of nominal demand ${ }_{0} E_{D} \equiv P^{0} r_{D}^{0}$ had before the Eurozone and with the rate of nominal demand before the

Euro crisis. We see ${ }_{3} E_{D}^{++}={ }_{0} E_{D}+P^{0} \delta_{D}+\frac{\delta_{I}}{\lambda_{S}}\left(r_{D}^{0}+\delta_{D}\right)$ or ${ }_{3} E_{D}^{++}={ }_{1} E_{D}+\frac{\delta_{I}}{\lambda_{S}}\left(r_{D}^{0}+\delta_{D}\right)$, i.e., the rate of nominal expanded demand increases relatively to the rates before both the Eurozone and the Euro crisis. The economic reforms bring good results here. The economic situation could last for a long time.

\subsection{Discussion and Interpretation}

We can briefly compare results of the pro-growth reforms in Greek economy obtained in both austere and benign economic conditions. It can be observed the austere conditions create a big hurdle in the form of the rate of external supply of products. In order to have an economic growth, Greek economy has to employ investment with the rate exceeding the rate of external supply of products. On the other hand, during the benign economic conditions when the rate of external supply of products is balanced via an increase of the 
demand the hurdle is nonexistent. Even an investment with small rate ${ }^{2}$ can produce an economic growth, which can be very beneficial for the Greek economy that experiences a deep economic recession.

\section{Conclusions}

A simplified economic model of the current Greek crisis is presented.

The theoretical attainment is that description of different stages of the crisis is done with a mathematical accuracy.

As a practical result, it is objectively shown that investment in the benign conditions of sustained demand produces a higher nominal economic growth than investment in the austere conditions of reduced demand.

\section{References}

Krouglov, Alexei, 2006, Mathematical Dynamics of Economic Markets (New York: Nova Science Publishers).

Krouglov, Alexei, 2009, "Mathematical Dynamics of Economic Growth as Effect of Internal Savings," Finance India, Vol. 23, No. 1, pp. 99-136.

Krouglov, Alexei, 2014, "Secular Stagnation and Decline: A Simplified Model." Available at SSRN: http://ssrn.com/abstract=2540408 or http://dx.doi.org/10.2139/ssrn.2540408.

Krouglov, Alexei, 2015a, "Credit Expansion and Contraction: A Simplified Model." Available at SSRN: http://ssrn.com/abstract=2604176 or http://dx.doi.org/10.2139/ssrn.2604176.

Krouglov, Alexei, 2015b, "Economic Growth and Debt: A Simplified Model." Available at SSRN: http://ssrn.com/abstract=2621227 or http://dx.doi.org/10.2139/ssrn.2621227.

Petrovski, Ivan G., 1966, Ordinary Differential Equations (Englewoods Cliffs, New Jersey: Prentice Hall).

Piskunov, Nikolai S., 1965, Differential and Integral Calculus (Groningen: P. Noordhoff).

\footnotetext{
${ }^{2}$ Of course, an investment with accelerated rate will produce best result as is shown in (Krouglov, 2014).
} 